\title{
Diffraction and Vector Mesons Working Group Summary
}

\author{
Markus Diehl ${ }^{1}$, Paul Laycock ${ }^{2}$ and Christophe Royon ${ }^{3}$ \\ 1- Deutsches Elektronen-Synchroton DESY \\ 22603 Hamburg, Germany \\ 2- University of Liverpool, Dept. of High Energy Physics \\ Oliver Lodge Laboratory, Liverpool L69 7ZE, UK \\ 3- CEA/IRFU/Service de Physique des Particules \\ 91191 Gif-sur-Yvette CEDEX, France
}

\begin{abstract}
We summarise the talks presented in the working group on diffraction and vector mesons at the DIS 2009 workshop.
\end{abstract}

\section{Experiment}

\subsection{Exclusive vector meson production and DVCS}

Exclusive vector meson production provides an ideal experimental testing ground for QCD, as the experimental signature is clean and a variety of theoretical calculations is available. A comprehensive review of exclusive vector meson production data at HERA was reported in 2, 3. Particular attention was drawn to the excellent agreement between the H1 and ZEUS collaborations on $\rho$ meson electro-production data, while the apparent discrepancies in the $\phi$ data may be understood at least qualitatively. The universality of vector meson production was addressed in particular in 2], where the conclusions are yet to be finalised, but certainly the data prefer a universal scale like $\frac{1}{4}\left(Q^{2}+M^{2}\right)$ rather than $Q^{2}$ and follow a power-law in the scaling variable.

The spin density matrix element analysis of these data exhibits a wealth of information, as reported in 3 . One hitherto unnoticed observation is that the double-flip amplitude may be non-zero, which may be the first hint for a nonzero gluon transversity distribution [4. The data exhibit a clear breaking of $s$-channel helicity conservation, which remains however a good approximation at low $t$. Generally, the kinematic dependences of both the cross sections and the spin density matrix elements are at least qualitatively understood in QCD. The data can be described using models based on generalised parton distributions (GPDs) or on the dipole approach, with gross features being described but differences in the detail.

Deeply virtual Compton scattering is a process with sensitivity to correlations of partons in the proton and has sensitivity to GPDs, as shown in [5]. Fig. 1a shows (top) the $Q^{2}$ dependence of a dimensionless variable $S$ related to the amplitude for the process with the $t$-dependence removed; (bottom) the $Q^{2}$ dependence of a variable $R$ related to the ratio of GPD to PDF. The data are precise enough to discriminate between GPD models and favour a full GPD model rather than one with only kinematical skewing.

Measured at H1, the exclusive production of photons at high momentum transfer $t$ at the proton vertex was presented in [6]. These data allow comparison of the experimental results with predictions based on a BFKL approach; thanks to the final state photon, these predictions do not suffer from uncertainty on the final state vector meson wave function. The gross features of the kinematic dependences of the data, i.e. the $W$ and $t$ dependences,

DIS 2009 
are well reproduced by the model. The $W$ dependence of the high- $t$ photon cross section is shown in Fig. 1b this is certainly one of the hardest diffractive processes yet measured, with an exponent $\delta=2.73 \pm 1.02$ (stat.) ${ }_{-0.78}^{+0.56}$ (sys.). This is consistent with the model predictions, although the precision of the data is limited experimentally by the small lever-arm in $W$.

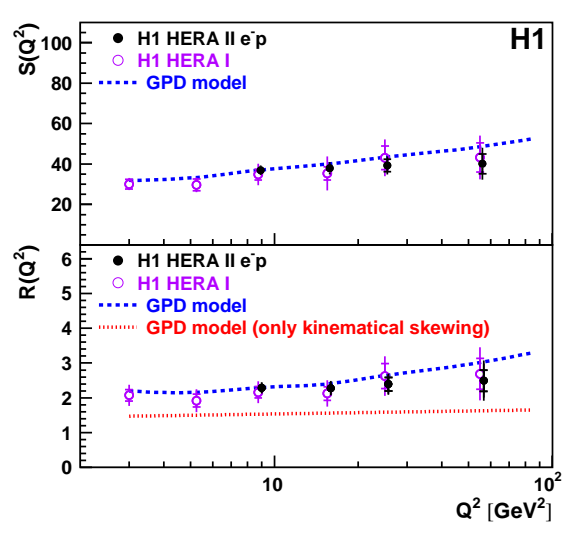

(a)

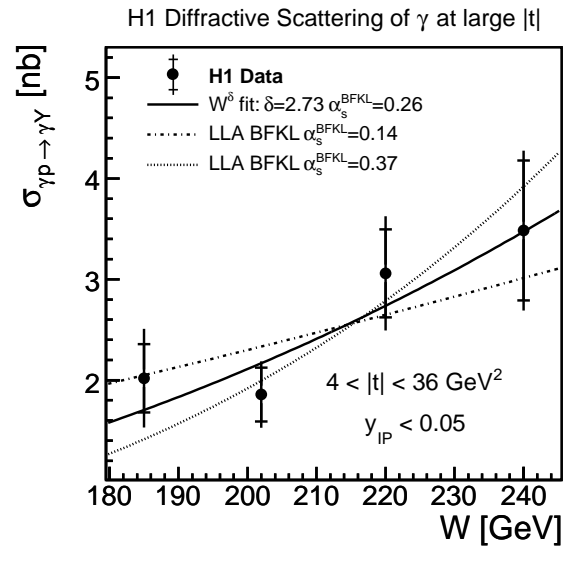

(b)

Figure 1: (a) The $Q^{2}$ dependence of quantities sensitive to GPDs (see text) and (b) the $W$ dependence of the high-t photon cross section.

The Pomeron trajectory in $\rho$ photo-production is one of the cleanest processes to measure experimentally and is assumed to be one of the cleanest to interpret theoretically, as it approaches the bare Pomeron. A new global fit to high precision $\mathrm{H} 1$ data at low $t$ combined with ZEUS and Omega data at higher $t$ was reported in [7. The global result, dominated by the low $t$ data, shows clear evidence of a non-linear trajectory; the high precision low $t$ data in particular show a marked departure from a straight line. Although this is not at all in disagreement with theory, it is an interesting result and in contradiction with the usual assumption of a linear trajectory.

Elastic $\rho$ production has also been studied by the COMPASS collaboration, where the asymmetry in angle between the target and hadron production planes is measured [8]. One key feature of the experimental setup is that three targets are used, allowing the asymmetry to be defined in such a way that the experimental effects completely cancel. The measured asymmetry is consistent with zero, in agreement with a previous measurement by the HERMES collaboration and also consistent with theoretical expectations based on GPD models.

A future measurement of DVCS at medium $x$ is planned at COMPASS, with high precision measurements of the $t$-slope of the process and the beam charge and spin asymmetry foreseen [9]. The promising results from a 2-day test run from 2008 show a detector performance consistent with the expectations of the technical design report.

The PHENIX collaboration have made a first measurement of the exclusive production of $\mathrm{J} / \Psi$ mesons in ultra-peripheral Au-Au collisions at RHIC, reported in [10]. This difficult measurement required a very good understanding of the significant irreducible continuum 


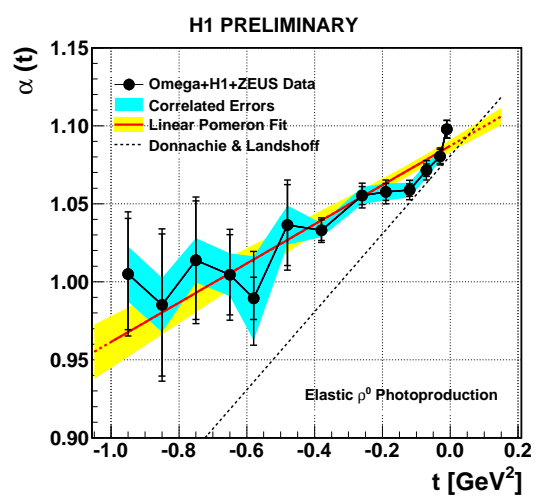

Figure 2: The Pomeron trajectory extracted from a global fit to $\rho$ photoproduction data.

background. The measured cross section agrees with theoretical predictions very well and, although the statistics at RHIC are limited, the agreement with expectation bodes very well for the LHC where this process will be measured in a new energy regime.

\subsection{Leading baryon production}

Leading neutrons have been studied by the PHENIX collaboration and the left-right production asymmetry measured, as reported in [11. A significant asymmetry is observed and both this asymmetry and the cross section measured differentially in longitudinal momentum fraction is consistent with models based on the pion exchange mechanism. Similar observations were reported by the ZEUS [12] and H1 [13] collaborations for large values of the longitudinal momentum of the neutron.

Leading protons have been studied by both the ZEUS [12] and H1 [14 collaborations, where the latter reported a new measurement on the full HERA II data set. The conclusions, especially on the proton vertex factorisation assumption, remain the same as for previous analyses, i.e. proton vertex factorisation holds within the large normalisation uncertainties which dominate the results. It is hoped that the Very Forward Proton Spectrometer (VFPS) of H1, installed for the HERA II period, will soon be able to shed more light on these issues with greater precision, thanks to its much larger acceptance.

\subsection{Forward detectors at the LHC}

Measurements of the total cross section and luminosity are foreseen in the ATLAS-ALFA [15] and TOTEM [16] experiments. Roman pots are installed at 147 and $220 \mathrm{~m}$ in TOTEM and at $240 \mathrm{~m}$ in ATLAS, and additional forward detectors in TOTEM called T1, T2 cover the rapidity regions $3.1<|\eta|<4.7$ and $5.3<|\eta|<6.5$. The measurement of the total cross section to be performed by the TOTEM collaboration [16] is shown in Fig. 3. We notice that there is a large uncertainty on predictions of the total cross section at the LHC energy due in particular to the discrepancy between the Tevatron measurements (CDF, E710 and E811). The measurement of TOTEM will be of special interest to solve that ambiguity. The 


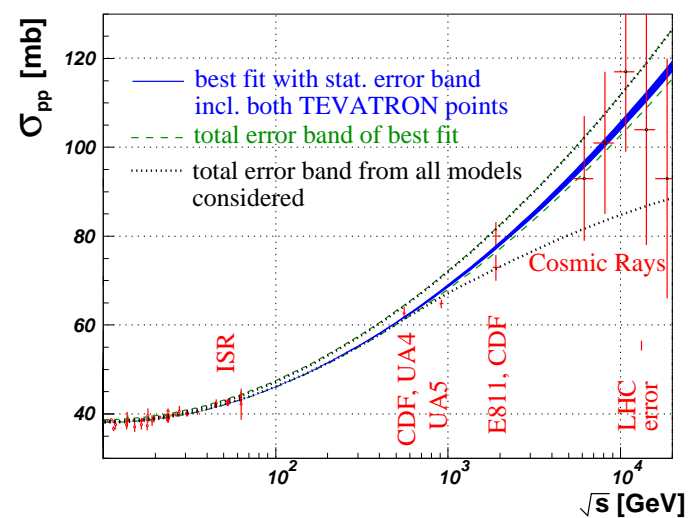

Figure 3: Measurement of the total $p p$ cross section.

TOTEM experiment will also allow to perform early measurements of single diffraction and double pomeron exchange using the horizontal detectors.

The ATLAS collaboration prefers to measure the elastic scattering in the Coulomb region [15], typically at very low $t\left(|t| \sim 6.5 \times 10^{-4} \mathrm{GeV}^{2}\right)$. When $t$ is close to 0 , the $t$ dependence of the elastic cross section reads:

$$
\left.\frac{d N}{d t}\right|_{t \rightarrow 0}=L \pi\left|\frac{2 \alpha_{\mathrm{em}}}{|t|}-\frac{\sigma_{\mathrm{tot}}}{4 \pi}(i+\rho) e^{-b|t| / 2}\right|^{2} .
$$

From a fit to the data in the Coulomb region, it is possible to determine directly the total cross section $\sigma_{\text {tot }}$, the $\rho$ and $b$ parameters as well as the absolute luminosity $L$. This measurement requires to go down to $t \sim 6.5 \times 10^{-4} \mathrm{GeV}^{2}$, or $\theta \sim 3.5 \mu \mathrm{rad}$ (to reach the kinematical domain where the strong amplitude equals the electromagnetic one). Once the absolute luminosity and the total cross section are known using these methods, the relative luminosity measurement as a function of time will be performed in ATLAS using the LUCID detector (Luminosity measurement Using Cerenkov Integrating Detectors) [15].

\subsubsection{Hard diffraction at the $\mathrm{LHC}$}

The LHC with a centre-of-mass energy of $14 \mathrm{TeV}$ will allow us to access a completely new kinematical domain in diffraction. The study of hard diffraction at the LHC will be performed using the rapidity gap method at low luminosity (without pileup) and the forward detectors at higher luminosity. With $10 \mathrm{pb}^{-1}$, about 300 single diffractive dijet events are expected while about 100 single diffractive $W$ events can be measured with $100 \mathrm{pb}^{-1}$. The correlation between the multiplicities in low and high $|\eta|$ (respectively $3.0<|\eta|<4.0$ and $4.0<|\eta|<5.0$ ) allows the isolation of diffractive events [17. The single diffractive measurements will determine the survival probability at LHC energies, as well as the rate and kinematic dependences of exclusive $\Upsilon$ photoproduction.

At higher luminosities, the CMS and ATLAS collaborations project to install forward detectors at 220-240 and $420 \mathrm{~m} \mathrm{[15,} \mathrm{18.} \mathrm{These} \mathrm{detectors} \mathrm{will} \mathrm{allow} \mathrm{the} \mathrm{study} \mathrm{of} \mathrm{hard}$ diffractive production of heavy objects such as the Higgs boson or the study of anomalous 
couplings between $\gamma$ and $W[18$. Two kinds of detectors namely 3D Si and timing detectors, will be hosted in movable beam pipes located at 220-240 and $420 \mathrm{~m}$. At the LHC, up to 35 interactions occur at the same bunch crossing; in order to determine which interaction a proton tagged in one of these detectors belongs to a timing resolution of better than 10-15 ps is needed. Lab results reported in [18] have demonstrated that this is indeed possible.

\subsection{Diffractive results from the Tevatron}

The CDF experiment at the Tevatron showed many new diffractive results [19. The main central CDF detectors (tracker and calorimeter) cover respectively the regions $|\eta|<2.0$ and

$|\eta|<3.6$. Beam shower counters and miniplug calorimeters extend the rapidity coverage between $5.4<|\eta|<7.4$ and $3.5<|\eta|<5.1$, respectively. Roman pot detectors were installed only on the $\bar{p}$ side and allow the detection of antiprotons with $0.02<\xi<0.1$ ( $\xi$ is the momentum fraction of the proton carried away by the pomeron). The extended coverage in rapidity using the miniplug calorimeters was especially important to reconstruct $\xi$ more precisely.

\subsubsection{Diffractive $W$ and $Z$ production}

The CDF collaboration measured the diffractive production of $W$ and $Z$ bosons, which probes the quark content of the pomeron. The fraction of $W$ events tagged in the Roman pot detectors is $0.97 \pm 0.05$ (stat.) \pm 0.11 (syst.) in the kinematical domain with $0.03<$ $\xi<0.1$ and $|t|<1 \mathrm{GeV}^{2}$, compatible with run I results, while the fraction of $Z$ events is $0.85 \pm 0.20$ (stat.) \pm 0.11 (syst.) in the same kinematical domain.

\subsubsection{Jet-gap-jet and Mueller-Navelet jets}

The miniplug calorimeters of the CDF collaboration allow a detailed study of rapidity gaps between jets (double diffractive events) as well as Mueller-Navelet jets as a test of BFKL resummation, which can be probed by measuring the difference in azimuthal angle between the two most forward jets [20]. The fraction of events with gaps is about $10 \%$ for soft double diffractive events and $1 \%$ for jet events with $E_{T}>2 \mathrm{GeV}$ in the miniplug calorimeter [19]. The $\Delta \eta$ dependence is found to be similar for both samples. It was reported in [21] that these measurements will also be possible at the LHC thanks to the very good coverage of the forward region by the ATLAS and CMS experiments.

\subsubsection{Inclusive diffraction at $C D F$}

A long-standing issue in the field of diffraction has been the complete breakdown of factorisation when diffractive PDFs from HERA are used to predict measurements at the Tevatron [22, 23. The CDF collaboration measured the ratio of dijet events in single diffractive and non-diffractive events. Assuming factorisation, this is directly proportional to the ratio of the diffractive to the inclusive proton densities. The CDF measurement is compared with a factorised calculation using some of the latest diffractive PDFs from H1 (Fit B and others) in Fig. 4, where a clear discrepancy of an order of magnitude can be seen. Understanding this discrepancy at a quantitative level is vital when making predictions for the LHC.

DIS 2009 


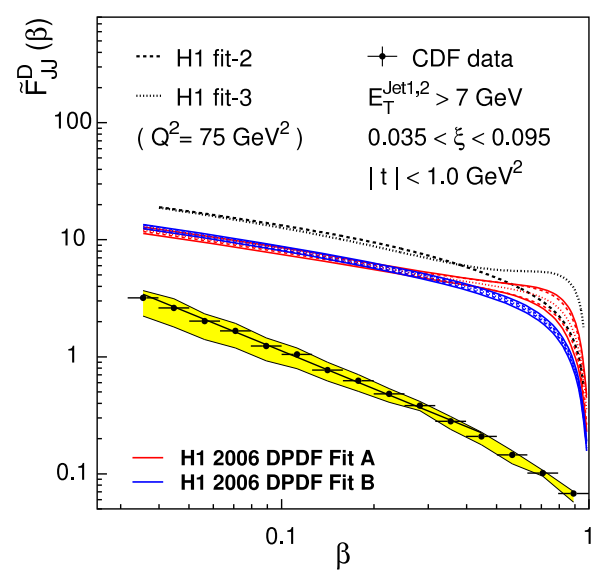

Figure 4: Comparison between the CDF measurement of diffractive dijet production (black points) with the expectation from factorisation using H1 fits of diffractive PDFs (the blue band shows Fit B).

\subsubsection{Search for exclusive events at the Tevatron}

Exclusive diffractive production, where the full energy is used to produce the final state object (Higgs boson, dijets, diphotons...), is a prominent topic in diffraction. Such events benefit from forward spectrometers with good energy resolution to provide a precise measurement of the mass of the final state object. CDF have looked for the presence of such events in double-pomeron exchange events, where the $\bar{p}$ is tagged and a rapidity gap is present on the other side [19]; the observable used is the dijet mass fraction. The comparison between the $\mathrm{CDF}$ data for a jet $E_{T}$ cut of $10 \mathrm{GeV}$ and the predictions from inclusive diffraction is given in Fig. 5 the data can only be described by the addition of a model of exclusive production.

Another interesting observable in the dijet channel is the rate of $b$ jets as a function of the dijet mass fraction. In exclusive events, the $b$ jets are suppressed because of the $J_{z}=0$ selection rule, and as expected, the fraction of $b$ jets in the diffractive dijet sample diminishes as a function of the dijet mass fraction [19].

Another way to look for exclusive events at the Tevatron is to search for the diffractive exclusive production of light particles like the $\chi, J / \Psi$ and $\Psi(2 S)$ mesons [19, 15]. Contrary to the diffractive exclusive production of heavy mass objects such as Higgs bosons, this leads to high enough cross sections to check the dynamical mechanisms and the existence of exclusive events. The CDF collaboration measured a $\chi$ production cross section of $\sim 75 \pm 14$ nb, compatible with theoretical expectations.

The CDF collaboration also looked for the exclusive production of diphotons 19. Two exclusive diphoton events have been observed by the CDF collaboration which is compatible with the expectations for exclusive diphoton production at the Tevatron. An update by the CDF collaboration with higher luminosity is expected very soon.

DIS 2009 


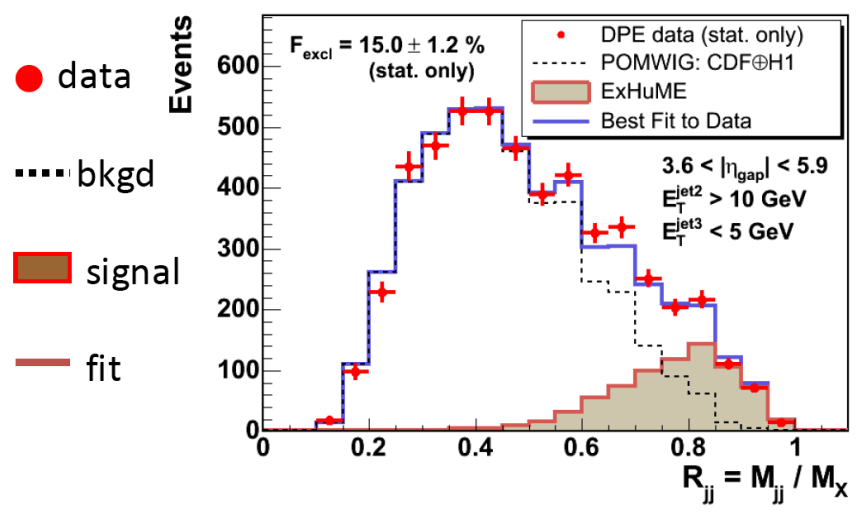

Figure 5: Dijet mass fraction measured by the CDF collaboration compared to the prediction adding the contributions from inclusive and exclusive diffraction.

\subsection{Factorisation and QCD fits}

The subject of rapidity gap survival probability can also be addressed by the HERA experiments [22, 23. Measurements of the ratio of diffractive dijets in photoproduction with respect to diffractive dijets in DIS can be used to see if the photon remnant, present in the resolved component of the photoproduction events, produces a suppression. Both $\mathrm{H} 1$ and ZEUS see no difference in the ratio of cross sections between a resolved-enriched sample of events and a direct-enriched sample. However, the two experiments seem to disagree on the value of the ratio itself, with H1 seeing clear evidence of a suppression of photoproduction events, while ZEUS measures a ratio compatible with there being no suppression. The current status on this issue is that both collaborations see evidence of an $E_{T}$ dependence (the transverse energy of the leading jet) of the ratio; given the different $E_{T}$ ranges of the two experiments, this could explain some of the apparent discrepancy. The normalisation uncertainty on the ratio is of the order of $20 \%$ and the ratio also depends on the choice of diffractive PDFs used in the NLO prediction. Further work towards consistency in these respects would help to clarify the current situation.

Another observable with potential sensitivity to rapidity gap suppression is the ratio of diffractive to inclusive dijets. This ratio has now been measured by the $\mathrm{H} 1$ collaboration, reported in 22. Unfortunately, the effect of the underlying event, modelled by multiple interactions (MI) in PYTHIA, also affect this ratio and are too large to be ignored. The MI model describes the data reasonably well, and the effect of including it or not yields a difference in the ratio of approximately the same size as is expected from suppression.

Despite the problems in understanding the photoproduction data, both H1 and ZEUS have shown that the diffractive dijet data in DIS agree very well with the factorisation assumption. Both collaborations have now made a combined fit to their inclusive and dijet DIS data, both yielding results in very good agreement with one another, as was reported in 23. Fig. [6 shows a summary plot of the latest published diffractive PDFs from the H1 Collaboration 24; the consistency between Fit B (without dijet data) and the H1 2007 Jets PDF which include the dijet data is good and gives us confidence that the NLO QCD

DIS 2009 


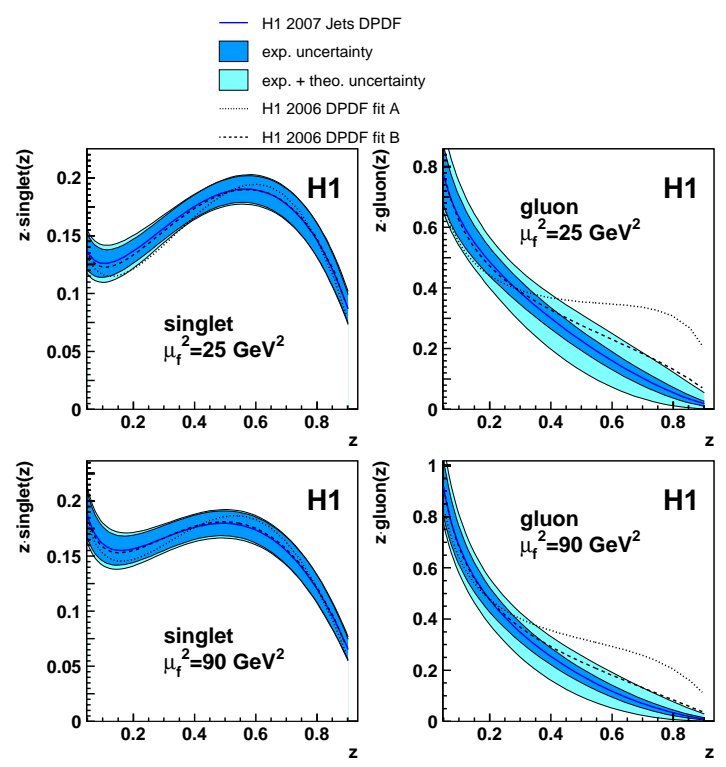

Figure 6: The H1 diffractive PDFs resulting from the combined fit to the inclusive and dijet diffractive DIS data.

picture used to determine these distributions is indeed appropriate.

\subsection{Inclusive diffraction}

The final analysis of inclusive diffractive data from ZEUS [25] using the large rapidity gap (LRG) method was reported in 26. The data, discussed in terms of the reduced cross section, are shown in Fig. 7 and compared to the published H1 LRG data and to a QCD fit to that data (Fit B). The precision of the ZEUS data across such a broad kinematic range is very impressive and the final measurements from H1 (using the full HERA II dataset) will be needed to make a meaningful combination.

\subsection{First measurement of $F_{L}^{D}$}

The reduced cross section contains both diffractive structure functions $F_{2}^{D}$ and $F_{L}^{D}$ and in most analyses is approximately equal to $F_{2}^{D}$ due to the $y$-dependent suppression factor:

$$
\sigma_{r}^{D}=F_{2}^{D}-\frac{y^{2}}{Y_{+}} F_{L}^{D}
$$

where $Y_{+}=1+(1-y)^{2}$. The longitudinal diffractive structure function, in analogy with the inclusive case, is approximately proportional to the diffractive gluon density, and a measurement of $F_{L}^{D}$ provides an independent cross check of the gluon density extracted from the scaling violations of $F_{2}^{D}$. Experimentally, the longitudinal structure function can be extracted from inclusive cross section measurements at different beam energies. Plotting $\sigma_{r}^{D}$ vs $y^{2} / Y_{+}$results in a straight line with slope $-F_{L}^{D}$. 

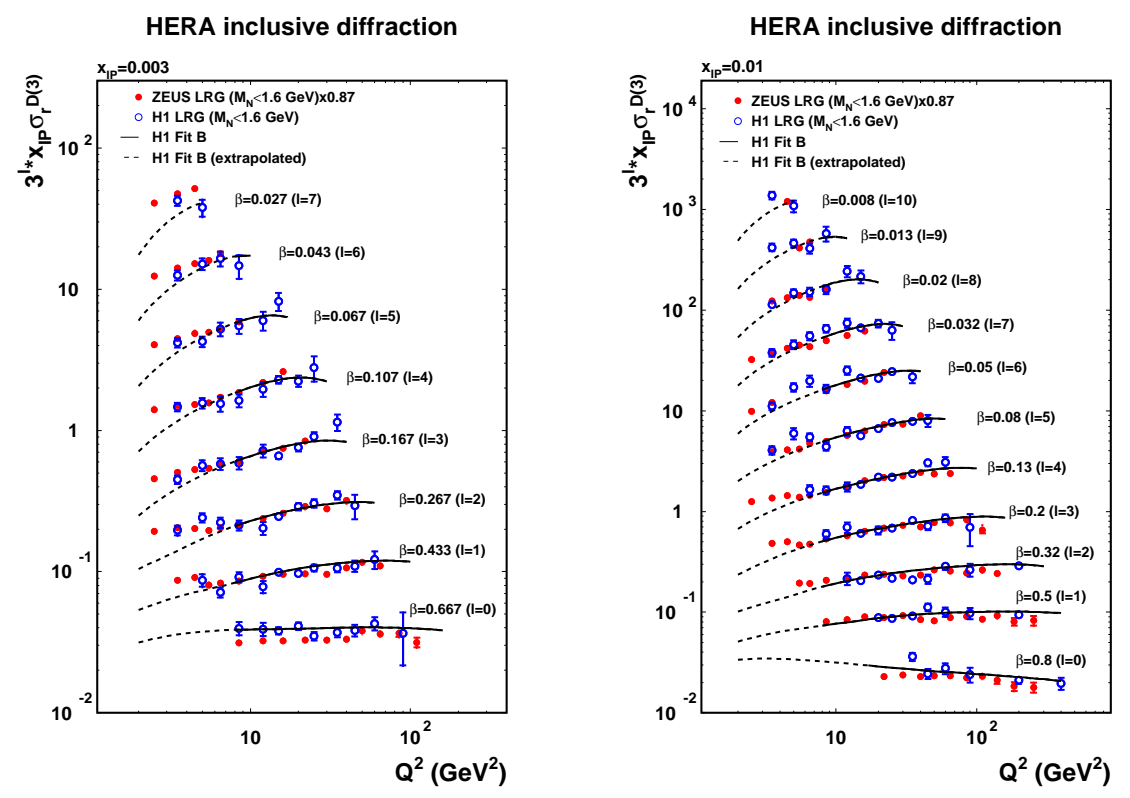

Figure 7: Comparison of the final ZEUS data on inclusive diffraction using the LRG method (red points) with the published H1 data (blue points) at two values of $x_{\mathbb{P}}$.

The final days of HERA running were devoted to two runs with proton beam energy different from $920 \mathrm{GeV}$, first at $460 \mathrm{GeV}$ and finally at $575 \mathrm{GeV}$. The measurement of $F_{L}^{D}$ using these data was reported in [27. It is a technically challenging measurement due to the need to measure at high values of $y$ (low scattered electron energy), resulting in a large irreducible photoproduction background that had to be understood in great detail.

The diffractive reduced cross sections at each beam energy are shown in Fig. 8a as a function of $\beta$. At high $\beta$ (low $y$ ) the reduced cross section is equal to $F_{2}^{D}$ and the data sets are normalised in this region to the result of H1 Fit B. The effect of $F_{L}^{D}$ can be seen at the lowest $\beta$ values as a suppression of the reduced cross section. Fig. $8 \mathrm{~b}$ shows the measured value of $F_{L}^{D}$ compared to the prediction of Fit B. The result is a confirmation of the NLO QCD picture of diffraction at greater than $3 \sigma$.

\section{Theory}

\subsection{The colour dipole formalism}

A large number of theory presentations in the working group were concerned with the colour dipole formalism. This formalism successfully describes a large variety of small- $x$ processes with a common non-perturbative input, namely the dipole scattering amplitude. Relevant observables are inclusive structure functions $\left(F_{2}, F_{L}, F_{2}^{c \bar{c}}\right)$, diffractive structure functions

$\left(F_{2}^{D}, F_{2}^{D(c \bar{c})}\right)$, and exclusive diffractive channels (vector meson production and DVCS). The colour dipole formulation is well suited to incorporate the dynamics of parton saturation, 


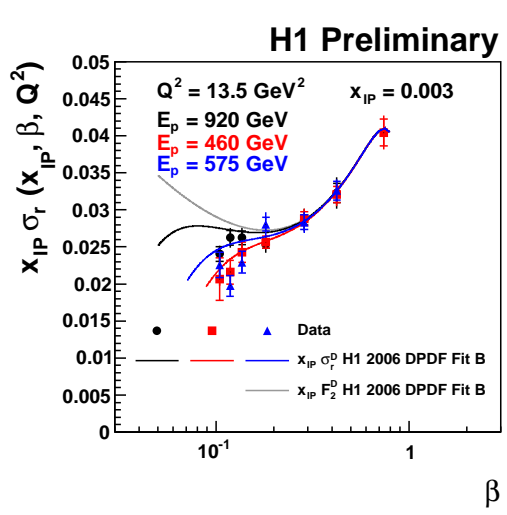

(a)

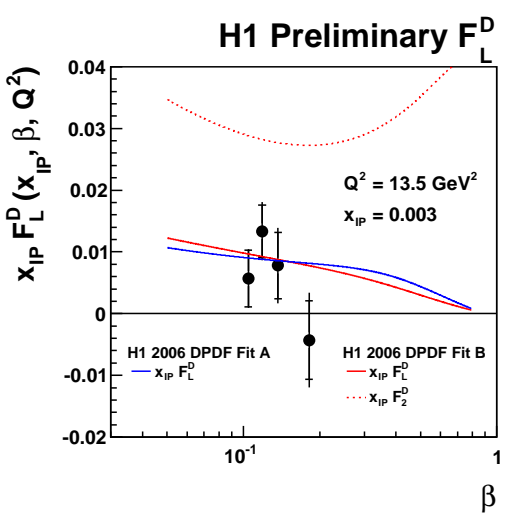

(b)

Figure 8: (a) The diffractive reduced cross section as a function of $\beta$ at three different beam energies. (b) The diffractive longitudinal structure function $F_{L}^{D}$ as a function of $\beta$.

with the dipole scattering amplitude entering in non-linear evolution equations (JIMWLK, Balitsky-Kovchegov, and their generalisations). Incorporated into the larger framework of the colour glass condensate, the colour dipole formulation provides a bridge between $e p$ scattering on one hand and $p p$ and heavy-ion collisions on the other [28.

To a large extent, the description of inclusive diffraction in DIS is based on two types of final states at parton level, as shown in Fig. 9. Complete theoretical expressions are available for the $q \bar{q}$ diffractive final state. It provides a scaling contribution to $F_{2}^{D}$ and a $1 / Q^{2}$ suppressed contribution to $F_{L}^{D}$ at large $\beta$. Its contribution to $F_{2, L}^{D(c \bar{c})}$ is nonzero but numerically negligible in typical HERA kinematics. By contrast, available calculations for the $q \bar{q} g$ final state are limited to either the leading $\log Q^{2}$ [29, 30, or the leading $\log (1 / \beta)$ [31] approximation. We note that in the latter case one has strong ordering between the longitudinal momenta of the final-state gluon and the $q \bar{q}$ pair, so that this configuration plays a role in JIMWLK evolution and requires particular care when applying this evolution to diffraction 32. An interpolation between the two logarithmic approximations has been devised and used in phenomenology 33. Unfortunately, both approximate results have only been obtained for light quarks. This is a rather unsatisfactory situation, because HERA measurements have shown that the fraction of charm in the diffractive final state can reach $20 \%$ to $30 \%$ [29, well above what is obtained from the $c \bar{c}$ final state in the dipole formulation. Analyses omitting diffractive charm altogether are hence severely limited in their precision, and it is difficult to assess the reliability of the different workarounds so far used in dipole-based descriptions.

A similar situation has emerged from the measurement of $F_{L}^{D}$ [27, which has been one of the experimental highlights in the diffractive session. The data points cover the $\beta$-range from 0.1 and 0.2 , where the contribution from the $q \bar{q}$ final state is completely negligible. As for the $q \bar{q} g$ state, the leading $\log (1 / \beta)$ approximation is not applicable in this region, whereas the leading $\log Q^{2}$ approximation gives a zero result This is clearly not good enough to

\footnotetext{
${ }^{\mathrm{a}} \mathrm{A} \log Q^{2}$ enhancement of the leading-twist contribution requires a nonzero leading-twist contribution at
} 

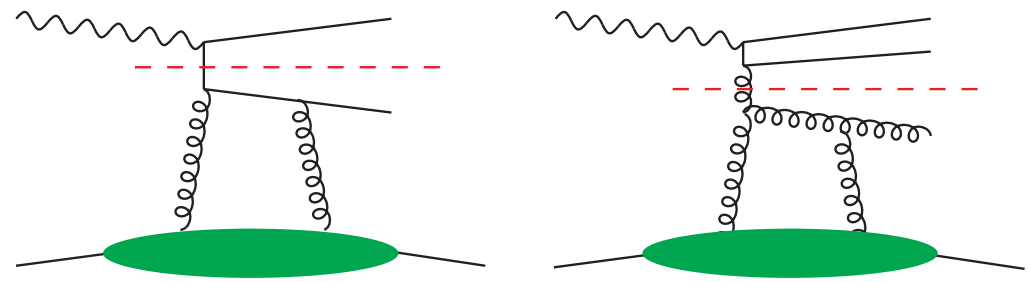

Figure 9: Graphs for the production of $q \bar{q}$ and $q \bar{q} g$ diffractive states in the colour dipole description. The horizontal dashed lines indicate the separation into a hard-scattering process and diffractive quark and gluon distributions in the large- $Q^{2}$ limit.

describe the existing data for $F_{L}^{D}$, and at the same time it may affect the accuracy for the description of $F_{2}^{D}=F_{T}^{D}+F_{L}^{D}$.

By now, analyses of HERA data within the dipole formulation typically address subtle questions such as the importance of parton saturation or the dependence on the impact parameter and its interplay with other kinematic variables. This requires sufficient precision of the theory, and in this respect the current data call for an improved theoretical evaluation of the $q \bar{q} g$ final state, for both light and heavy quarks.

It has been realised long ago [34 that the dipole formulation for $q \bar{q}$ and $q \bar{q} g$ final states can be used to obtain a simple prediction for diffractive PDFs, using suitable approximations of the dipole results in kinematics where diffractive PDFs can be used. The resulting PDFs pertain to a low scale; DGLAP evolution then accounts for further radiation leading to final states $q \bar{q} g g$ etc. This idea has been revived in a recent study [29], where the diffractive PDFs extracted from a dipole model were compared with those obtained in a DGLAP fit to $F_{2}^{D}$. Quite good agreement was found for the diffractive quark distribution at momentum fractions $z>0.3$, where the generation of low-momentum sea quarks by evolution does not play a major role. In turn, a clear discrepancy was observed for the diffractive gluon density $g^{D}(z)$, with the distribution extracted from the dipole model vanishing smoothly for $z \rightarrow 1$, whereas the DGLAP fit gave a pronounced peak at high $z$ for moderate factorisation scales.

This should, however, not be regarded as a shortcoming of the dipole result. Confirming earlier findings, a recent dedicated analysis [35] has emphasised that $F_{2}^{D}$ data alone provide only a poor constraint on $g^{D}(z)$ at high $z$. (An important result of the same study is that even within the resulting uncertainty, the diffractive gluon density is not sufficiently large at high $z$ to account for the rate of exclusive diffractive dijets observed by CDF [19]. This underlines the need for a genuinely exclusive production mechanism.) Furthermore, the data for diffractive dijet production clearly disfavour a pronounced bump of $g^{D}(z)$ at high $z$ compared with a smooth drop to zero 22 . From this perspective, the dipole model result for $g^{D}(z)$ in 29 seems to be in line with DGLAP phenomenology.

An important property of the dipole scattering amplitude is its dependence on the impact parameter $b$. It describes the spatial distribution of colour charge in the target and is of particular importance in the context of saturation, which will set in earlier in the dense centre of a hadron than at its more dilute edge. The $t$ dependence of inclusive and exclusive diffractive cross sections measured at HERA plays an essential role in attempts to determine

one order lower in $\alpha_{s}$, i.e. from the $q \bar{q}$ state. Such a contribution is present for $F_{T}^{D}$, but not for $F_{L}^{D}$. 
the $b$ dependence of the dipole scattering amplitude. A non-trivial pattern of preferred $b$ values in diffractive $q \bar{q}$ and $q \bar{q} g$ states has been discussed in [33], and characteristic predictions for inclusive diffraction on nuclei have been deduced, with a suppression at low and an enhancement at high $\beta$ compared to the proton case. As to exclusive diffraction on nuclei, detailed studies for vector meson production and DVCS have been given in [36, 37, with emphasis on the differences between the elastic contribution and nuclear breakup.

\subsection{Generalised parton distributions and exclusive diffraction}

The concept of generalised parton distributions (GPDs) permits the description of exclusive processes in a wide kinematic regime, and it offers the possibility of three-dimensional imaging of partons in the nucleon or in nuclei. An impressive amount of relevant data has been taken both in fixed-target experiments [38] and in the small- $x$ regime of H1 and ZEUS [2, 3]. There are encouraging prospects of measuring exclusive $J / \Psi$ and $\Upsilon$ photoproduction on the proton or nuclei at the LHC 10, which would extend existing measurements of these theoretically rather clean channels to very high energies. Additionally, ultraperipheral collisions at LHC offer the opportunity to study timelike Compton scattering, $\gamma p \rightarrow \ell^{+} \ell^{-} p[39$. Comparison of this reaction with DVCS at small $x$ measured at HERA would permit a key test for the theory description, which is closely related for the underlying QCD processes $\gamma^{*} p \rightarrow \gamma p$ and $\gamma p \rightarrow \gamma^{*} p$ (with a spacelike photon in one and a timelike photon in the other

case). Finally, generalised parton distributions are an important input to the description of exclusive diffraction in $p p$ collisions at the LHC [40].

The clarification of a long-standing theoretical dispute has been reported in the working group. In an early paper, Shuvaev et al. 41] claimed that in the small- $x$ limit, GPDs at $t=0$ can be calculated from the usual parton densities. A simple formula was derived for the case where the PDFs follow a power-law in $x$ and has been used in many phenomenological analyses. It is now clear [42, 43] that this claim cannot be upheld. The original derivation in [4]. fails due to subtleties in an inverse moment transform (which generalises the inverse Mellin transform for conventional PDFs), and there exist explicit examples of GPDs that satisfy all known symmetry relations but contradict the Shuvaev formula. Additional properties are required to ensure the validity of this formula, which given our present understanding should be understood as an ansatz, to be checked against data. A detailed study of this issue for both quark and gluon distributions has been reported in [42. At the same time, the Shuvaev ansatz can readily be used for PDFs that do not exhibit a power behavior, and a corresponding numerical code is available [43].

Exclusive Higgs production has long been considered a highlight for diffractive physics at the LHC. Its potential to explore physics beyond the standard model has been reviewed in 44, with emphasis on the minimal supersymmetric Standard Model. On the stronginteraction side, a major open issue remains the evaluation of the rapidity-gap survival probability for exclusive diffraction in LHC kinematics; a topic that merits additional attention due to its connection with the physics of multiple parton interactions [45. The assessment presented in [40] is that interactions involving hard spectator partons may be more important than assumed in most present estimates. This effect would increase with energy and could decrease the survival probability at the LHC without affecting the corresponding estimates for Tevatron kinematics, which are consistent with the observed rate of exclusive diffractive dijets [19. More detailed theoretical work will be needed to clarify this issue.

DIS 2009 


\subsection{Further theoretical developments}

Theoretical progress on many more aspects of diffraction has been reported in the working group and can only be briefly mentioned in this summary.

Work on a unified description of diffraction with either an intact final-state proton or with proton dissociation was reported in [37, with the aim of treating diffractive measurements at low and high $t$ in a common formalism based on the colour glass condensate. An analysis of Mueller-Navelet jets and rapidity gaps between jets in the BFKL framework was presented in [20]. NLO corrections to the BFKL kernel were found to be moderate, and a comparison with Tevatron data was given, as well as predictions for the LHC.

The exclusive production of forward jets, $p p \rightarrow p+3$ jets was explored in the $k_{T}$ factorisation framework [46. This process is sensitive to the $k_{T}$ unintegrated gluon GPD in the proton on one side and the three-quark proton distribution amplitudes on the other, and it will be interesting to see whether it can be measured with forward detectors at the LHC. The process $\gamma \gamma \rightarrow\left(\pi^{+} \pi^{-}\right)+\left(\pi^{+} \pi^{-}\right)$was investigated in [47, where it was shown that appropriate charge asymmetries in the final state project out the interference between perturbative odderon and pomeron exchange. Estimates indicate that the asymmetry may be large, but more detailed experimental studies will be required to establish whether this channel can be observed in ultraperipheral $p p$ or heavy-ion collisions at the LHC.

Various extensions of the Standard Model predict new heavy strongly-interacting particles. Some of these would be metastable and hence form bound states with ordinary quarks or gluons, before decaying inside the LHC detectors. The interactions of such heavy hadrons with the detector material were studied in [48. An important finding was that in certain cases an initially charged hadron tends to be converted to a neutral one by repeated scattering, which is of immediate consequence for experimental search strategies.

\section{Conclusions}

Although in general we see that the gross features are understood in QCD, there remain many questions to be answered in the fields of diffractive and vector meson physics. The issue of rapidity gap survival probability may be qualitatively understood, but the experimental status of the subject at HERA remains less than ideal. Nevertheless, the experimental results from the Tevatron on exclusive production agree well with theory and bode well for extrapolating our current understanding to the LHC domain.

The HERA data have helped to elucidate, confirm and reject much; the measurement of $F_{L}^{D}$ confirms that NLO QCD is applicable to inclusive diffraction in DIS. The exclusive vector meson and DVCS results can be understood in terms of models based on either GPDs or the colour dipole approach. Nevertheless, precision can still be improved here, and the final publications from $\mathrm{H} 1$ and ZEUS as well as data combinations are eagerly anticipated and needed. These, together with the promising physics program of the VFPS of H1, mean that the final word from HERA has not yet been uttered.

\section{Acknowledgments}

We would like to thank all speakers in the working group for their carefully prepared contributions, our co-convenors from the working groups on Structure Functions and low- $x$ and

DIS 2009 
on Spin Physics for organising common sessions, and J. Terron, C. Glasman, A. Sabio Vera and C. Uribe-Estrada for hosting a wonderful meeting.

\section{Bibliography}

\section{References}

[1] Slides:

http://indico. cern. ch/contributionDisplay . py? contribId=327\&sessionId=22\&conf Id=53294

http://indico. cern. ch/contributionDisplay. py? contribId=\&sessionId=22\&conf Id=53294

[2] A. Levy, these proceedings.

[3] P. Marage, these proceedings.

[4] N. Kivel, Phys. Rev. D 65 (2002) 054010 arXiv:hep-ph/0107275.

[5] F. D. Aaron et al. [H1 Collaboration], Phys. Lett. B 659 (2008) 796 arXiv:0709.4114 [hep-ex]].

[6] T. Hreus, these proceedings.

[7] B. List, these proceedings.

[8] G. Jegou, these proceedings.

[9] E. Burtin, these proceedings.

[10] M. Csanád, these proceedings.

[11] M. Togawa, these proceedings.

[12] G. Bruni, these proceedings.

[13] V. Dodonov, these proceedings.

[14] M. Kapishin, these proceedings.

[15] J. Pinfold, these proceedings.

[16] H. Niewiadomski, these proceedings.

[17] M. Obertino, these proceedings.

[18] K. Piotrzkowski, these proceedings.

[19] C. Mesropian, these proceedings.

[20] F. Chevallier, these proceedings.

[21] B. Roland, these proceedings.

[22] P. Newman, these proceedings.

[23] W. Słominski, these proceedings.

[24] A. Aktas et al. [H1 Collaboration], Eur. Phys. J. C 48 (2006) 715 arXiv:hep-ex/0606004];

A. Aktas et al. [H1 Collaboration], JHEP 0710, 042 (2007) arXiv:0708.3217 [hep-ex]].

[25] S. Chekanov et al. [ZEUS Collaboration], Nucl. Phys. B 816, 1 (2009) arXiv:0812.2003 [hep-ex]].

[26] M. Ruspa, these proceedings.

[27] D. Šalek, these proceedings.

[28] N. Armesto, these proceedings.

[29] A. Łuszczak, these proceedings.

[30] M. Wüsthoff, Phys. Rev. D 56 (1997) 4311 arXiv:hep-ph/9702201.

[31] J. Bartels, H. Jung and A. Kyrieleis, Eur. Phys. J. C 24 (2002) 555 arXiv:hep-ph/0204269;

S. Munier and A. Shoshi, Phys. Rev. D 69 (2004) 074022 arXiv:hep-ph/0312022.

[32] H. Weigert, these proceedings.

[33] T. Lappi, H. Kowalski, C. Marquet and R. Venugopalan, these proceedings arXiv:0906.3637.

[34] W. Buchmüller, T. Gehrmann and A. Hebecker, Nucl. Phys. B 537 (1999) 477 arXiv:hep-ph/9808454.

DIS 2009 
[35] L. Schoeffel, these proceedings arXiv:0906.2463.

[36] M. Machado, these proceedings.

[37] C. Marquet, these proceedings.

[38] C. Gagliardi, R. Sassot and G. Schnell, these proceedings.

[39] B. Pire, L. Szymanowski and J. Wagner, these proceedings arXiv:0906.5256].

[40] C. Weiss, these proceedings.

[41] A. G. Shuvaev, K. J. Golec-Biernat, A. D. Martin and M. G. Ryskin, Phys. Rev. D 60 (1999) 014015 arXiv:hep-ph/9902410.

[42] K. Kumerički and D. Müller, these proceedings arXiv:0907.1207.

[43] T. Teubner, these proceedings.

[44] V. A. Khoze, these proceedings.

[45] J. Bartels, these proceedings.

[46] D. Yu. Ivanov, these proceedings.

[47] B. Pire, F. Schwennsen, L. Szymanowski and S. Wallon, these proceedings arXiv:0906.5512.

[48] D. Milstead, these proceedings. 